\title{
Evaluation de la fertilité des sols et les rendements de cotonnier, maïs et sorgho à Gliricidia sepium (Jacq.) Kunth ex. Walp
}

\author{
Salif DOUMBIA ${ }^{1 *}$, Sidiki G. DEMBELE ${ }^{2}$, Fagaye SISSOKO $^{1}$, Odiaba SAMAKE ${ }^{1}$, \\ Fernando SOUSA ${ }^{3}$, Harun CICEK ${ }^{3}$, Noah ADAMTEY ${ }^{3}$ et Andreas FLIESSBACH ${ }^{3}$

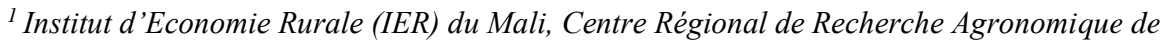 \\ Sikasso. BP 16, Sikasso, Mali. \\ ${ }^{2}$ Institut Polytechnique Rural de Formation et de Recherche Appliquée (IPR/IFRA). BP 06, Koulikoro, Mali. \\ ${ }^{3}$ Research Institute of Organic Agriculture, Department of Soil Sciences, Ackerstrasse 113, CH-5070 Frick, \\ Suisse. \\ *Auteur correspondant ; E-mail: doumbiasalif@gmail.com / salif.doumbia@ier.ml, \\ Tél : +22363032220/76258708
}

\section{REMERCIEMENTS}

Cette étude a été réalisée dans le cadre des activités du projet "Farmer driven organic resource management to build soil fertility (ORM4Soil) au Mali. Les auteurs remercient le Programme suisse de recherche sur les enjeux mondiaux du développement (programme r4d), lancé par la Direction du Développement et de la Coopération (DDC) et le Fonds National Suisse (FNS) pour le financement du projet.

\section{RÉSUMÉ}

De nos jours, la carence en fertilité des sols est un problème très répandu dans la plupart des pays subsahariens. G. sepium a été testé en association avec le cotonnier, le maïs et le sorgho afin de développer les meilleures pratiques dans deux stations de recherche et en milieu paysan dans les zones soudaniennes et soudanoguinéenne du Mali. L'objectif de cette étude était de développer, avec les producteurs participants, une technologie agro-forestière basée sur le Gliricidia sepium dans le système de culture en couloir. Le dispositif expérimental utilisé a été le Split plot à quatre répétitions. Le pH, le carbone organique du sol et l'azote ont été analysé dans des échantillons de sol de 0 à $20 \mathrm{~cm}$ de profondeur prélevés en 2016 au début de l'expérience et en 2018. Les rendements grains moyens de coton, de maïs et de sorgho associés au G. sepium étaient respectivement de 812, 1457 et $1377 \mathrm{~kg} \cdot \mathrm{ha}^{-1}$, alors qu'ils étaient significativement plus faibles sans G. sepium, avec 623, 1014 et 807 kg.ha ${ }^{-1}$ respectivement. Pendant la troisième année suivant la plantation de G. sepium, la teneur en carbone organique et en azote total du sol a augmenté de $5 \%$ dans les sols situés entre les allées. Le G. sepium en association avec les cultures a le potentiel d'améliorer leurs rendements et le sol.

(C) 2020 International Formulae Group. All rights reserved.

Mots clés : Carbone organique, azote, rendements, zones soudanienne et soudano-guinéenne, Mali

\section{Evaluation of Gliricidia sepium (Jacq.) Kunth ex. Walp effect on soil fertility, yields of cotton, maize and sorghum}

\begin{abstract}
Nowdays, soil fertility deficiency is a widespread issue in most Sub-Saharian countries specially where the application of less input is the common agricultural practices. Thus the legume plant, G. sepium was established with cotton, maize and sorghum to develop best practices for increasing crop yield and soil fertility
\end{abstract}


at two research stations and farming environment in the Sudanian and Sudano-Guinean zones of Mali. The objective of this study was to develop an agroforestry technology with farmers based on Gliricidia sepium in alley cropping system. The experimental design was Split plot with four replications. Soil pH, soil organic carbon and nitrogen were analyzed from 0-20 cm deep soil samples taken in 2016 at the beginning of the experiment and in 2018. Mean grain yields of cotton, maize and sorghum associated with G. sepium were 812, 1457 and $1377 \mathrm{~kg} . \mathrm{ha}^{-1}$, respectively, while they were significantly lower without G. sepium with 623, 1014 and 807 kg.ha- ${ }^{-1}$ as respectively cotton, maize and sorghum yield. In the third year after planting G. sepium, the soil organic carbon and total nitrogen content increased by $5 \%$ between the alleys. G. sepium in association with crops has the potential to improve crop yields and soil fertility.

(C) 2020 International Formulae Group. All rights reserved.

Keywords: Gliricidia sepium, organic carbon, nitrogen, yields, Mali.

\section{INTRODUCTION}

Le secteur agricole est l'épine dorsale de l'économie des pays du Sahel. Environ 80\% de la population de ces pays vit dans des zones rurales et dépend de l'agriculture, de la pêche et de l'exploitation des ressources forestières pour sa subsistance. Les effets conjugués de pratiques agricoles inappropriées et des effets négatifs du changement climatique menacent les moyens de subsistance des populations rurales du Sahel. La productivité des sols diminue et les terres arables se dégradent, les terres disponibles pour le pâturage se réduisent, avec pour conséquence l'insécurité alimentaire et des États déstabilisés et des conflits, non seulement entre agriculteurs et éleveurs (Konaté, 2008). Au Mali, la dégradation des sols touche environ $22 \%$ des terres arables, ce qui est devenu une préoccupation majeure pour la production agricole (FAO et al., 2015). Selon Jones et al. (2013), ce problème est lié, entre autres, à l'utilisation de terres moins productives ou marginales, à des périodes de jachère plus courtes, au surpâturage et à la surutilisation du couvert végétal, qui entrainent une dégradation des sols (c'est-à-dire une baisse de la fertilité et une acidification des sols).

$\mathrm{Au}$ cours des dernières décennies, l'augmentation de la productivité au Mali a été obtenue principalement par l'expansion des terres cultivées et l'exploitation des sols par l'enlèvement continu des nutriments du sol sans compensation. Par exemple, la superficie consacrée au maïs est passée de 59247 ha en 2008 à 298150 ha en 2017 (DNA, 2017). De même, la surface cultivée en cotonnier est passée de 28360 ha à 703652 ha entre 1960 et 2017 (CMDT, 2018). Au cours de cette période, la population malienne a été multipliée par quatre, pour atteindre environ 19 millions de personnes. Ainsi, le gouvernement malien est confronté au défi de produire des denrées alimentaires pour nourrir une population en constante augmentation tout en préservant les ressources naturelles. Les bilans nutritionnels des systèmes de culture du cotonnier au Mali sont négatifs et les niveaux de macronutriments dans les sols sont inférieurs aux seuils critiques de $300 \mathrm{mg} \mathrm{N} \mathrm{g}^{-1}$ sol, 2,2 à 5,5 mg $\mathrm{P} \mathrm{kg}^{-1}$ et 0,01 à 0,07 meq $\mathrm{K} 100 \mathrm{~g}^{-1}$ (Dembele et al., 2016). Il a été démontré que l'ajout d'amendements organiques dans ces sols dégradés augmente la matière organique du sol, le $\mathrm{pH}$, la capacité de rétention d'eau et la productivité globale (Bayala et al., 2014). Les avantages de l'intégration des arbustes et du stress dans les systèmes de culture sahéliens sont bien connus, mais une compréhension scientifique approfondie ainsi que l'adoption et la mise en œuvre à grande échelle de tels systèmes sont rares (Lahmar et al., 2012 ; Bayala et al., 2014). De même, les avantages des arbustes et des arbres qui fournissent des services écosystémiques (contrôle de l'érosion, refuge pour la biodiversité, séquestration du carbone, ombrage, nourriture/fourrage et médicaments pour les animaux et les humains) et qui ont une importance culturelle pour la population sont largement reconnus (Bado et al., 2016). En Afrique de l'Ouest, les ligneux contribuent à la satisfaction des besoins en aliments, en bois d'énergie, en bois d'œuvre, en bois de service et en phytothérapie de la majorité des 
populations (Traoré et al., 2011). Aussi, la conservation de la biomasse en couverture sur le sol permet de mieux conserver l'humidité (Ouattara et al., 2018). Malgré les preuves, un certain nombre de technologies de gestion de la fertilité des sols sont mal adoptées en raison de contraintes biophysiques, économiques ou socioculturelles ( Maraux et al., 2007). Il est impératif d'introduire des outils et des technologies de gestion de la fertilité des sols qui soient abordables pour les producteurs à faibles revenus et respectueux de l'environnement. Gliricidia sepium est l'une de ces espèces arbustives qui peut être intégrée dans les systèmes à base de maïs et de cotonnier, avec une concurrence négligeable à fournir une quantité élevée d'azote au système (Partey et al., 2018) et à augmenter la matière organique du sol (Beedy et al., 2010), ce qui est particulièrement pertinent pour les régions où la disponibilité et le coût des amendements organiques ou inorganiques sont limités. L'objectif de cette étude était de développer, avec les producteurs participants, une technologie agro-forestière basée sur le Gliricidia sepium dans le système de culture en couloir pour aider à améliorer les sols, la productivité des cultures et la sécurité alimentaire dans la ceinture du Sahel au Mali.

\section{MATÉRIEL ET MÉTHODES Processus participatif}

En 2016, une étude diagnostique a été conduite sur le terrain, dans les deux sites d'étude choisis (Zoumana Diassa dans les régions de Sikasso et Mafeya dans la région de Koulikoro), l'équipe de recherche interdisciplinaire a contacté que les producteurs ont été ensemble convenu de plusieurs approches pour améliorer la fertilité des sols. Ils ont discuté de plusieurs techniques qui pourraient être utiles pour réduire les coûts et les intrants ainsi que pour stabiliser les rendements en améliorant la fertilité des sols. Les participants ont convenu que les systèmes agroforestiers étaient essentiels pour a) la fertilité des sols, en raison des nombreux avantages qu'ils procurent ; b) la sécurité alimentaire, en améliorant le rendement des cultures, en réduisant les coûts des engrais et en servant de source de fourrage pour le bétail pendant la saison sèche ; et c) l'adaptation au climat, en protégeant les sols et en accumulant de la matière organique. Les inconvénients ont également été discutés, tels que l'augmentation du travail manuel aux heures de pointe pour la gestion des couloirs et la perte de terres couvertes par les allées. Comme les producteurs n'utilisent pas d'herbicides, cela n'a pas empêché d'inclure plus d'une espèce dans le champ. Au final, 15 tests sur le terrain dans les exploitations agricoles et une expérimentation en station ont été installés dans chacune des deux régions.

\section{Présentation des sites d'étude}

Les systèmes agroforestiers avec des couloirs de G. sepium ont été testés en 2017 et 2018 dans les stations de recherche de l'IPR/IFRA de Katibougou, dans la région de Koulikoro et de l'IER dans la sous-station de Farako au Centre Régional de Recherche Agronomique de la région de Sikasso (Tableau 1). Les groupes de producteurs respectifs se trouvaient dans les villages de Mafèya (Koulikoro) et de Zoumana Diassa (Sikasso). La région de Sikasso a reçu environ $30 \%$ de précipitations de plus que la région de Koulikoro (Figure 1). Les précipitations ont été relativement faibles à Katibougou par rapport à Farako. En particulier en 2017 à Katibougou, la quantité de pluie et sa répartition n'étaient pas appropriées pour donner de bonnes conditions de croissance.

\section{Matériel}

Selon les zones agro-écologiques et les séquences de rotation des cultures, des variétés adaptées de sorgho, de maïs et de cotonnier ont été utilisées. Dans les deux stations d'expérimentation, les traitements ont inclus la biomasse de G. sepium (Jacq.) Kunth ex. Walp., variété ILG50 (de l'ICRAF/Mali) en culture intercalaire avec du cotonnier (Gossypium hirsutum L., STAM59A) et du maïs (Zea mays L. hybride Dembagnuma) à Sikasso et en culture intercalaire avec du sorgho (Sorghum bicolor L.) variété "CSM 63 E" et du maïs (Zea mays L. hybride "brico") à Koulikoro. 


\section{Caractéristiques des sols dans les stations}

Les résultats des analyses de sol au début des expérimentations montrent que le sol du site de Farako dans la région de Sikasso est sableux et que celui de Katibougou est limoneux sableux (Tableau 2). Les sols sont légèrement acides avec des valeurs de $\mathrm{pH}$ de 5,4 et sont tous deux très pauvres en matière organique du sol, en azote total du sol, en P et en $\mathrm{K}$ comme exemples de nutriments pour les plantes. C'est une caractéristique typique des sols de la région lorsqu'ils sont utilisés pour la production de cultures pendant une longue période.

\section{Apports de nutriments}

L'engrais minéral a été appliqué conformément aux recommandations officielles pour les différentes cultures (Tableau 3). Le maïs et le sorgho ont été fertilisés avec un engrais complexe céréalier $15 \mathrm{~N}-15 \mathrm{P}-15 \mathrm{~K}$ au moment du semis et de l'urée 45 jours après la levée. Le cotonnier a été fertilisé avec un engrais complexe de coton $14 \mathrm{~N}-10 \mathrm{P}-10 \mathrm{~K}-7 \mathrm{~S}-1 \mathrm{~B}, 15$ jours après le semis et de l'urée 45 jours après la germination. Le compost a été préparé dans une fosse de $3 \times 3$ $\mathrm{m}$ et $1 \mathrm{~m}$ de profondeur, progressivement remplie de paille, de résidus de culture, d'ordures ménagères et de déchets animaux. Il a été maintenu humide et a été retourné tous les 15 jours au moins 4 fois. Le compost était appliqué à raison de $5 \mathrm{t} \mathrm{ha}^{-1}$ (Dose recommandée) et de $2,5 \mathrm{tha}^{-1}$ (Demi-dose) tous les 2 ans. Les producteurs ont apporté le compost sur les parcelles avant le labour en mai et juin. L'engrais minéral a été appliqué selon la recommandation officielle aux cultures sous la forme suivante : Pour le maïs et le sorgho, $100 \mathrm{~kg} \mathrm{ha}^{-1} \mathrm{~d}^{\prime}$ engrais complexe (15P-15P-15K) et 150 et $50 \mathrm{~kg} \mathrm{ha}^{-1}$ d'urée respectivement. Pour le cotonnier, $200 \mathrm{~kg} \mathrm{ha}^{-1}$ d'engrais complexe pour le cotonnier (14N-22P-12K-7S-1B) et 50 $\mathrm{kg} \mathrm{ha}^{-1}$ d'urée $(46 \% \mathrm{~N})$. La biomasse de $G$. sepium provenant de la taille a été collectée et répartie de manière égale sur les parcelles respectives avec des allées de G. sepium par la suite (Tableau 4).

\section{Le dispositif expérimental}

Le dispositif expérimental utilisé a été le Split plot avec deux systèmes de culture (avec (+G) et sans G. sepium $(-G)$ ) comme facteur principal et cinq traitements d'engrais comme facteur secondaire et reproduits quatre fois. Les traitements fertilisants comprenaient un témoin Sans Fertilisation Minérale (SFM), deux traitements avec la Fertilisation Organique : la Fertilisation Organique Recommandée (FOR) et la demi-dose $(1 / 2 \mathrm{FOR})$, et deux traitements avec la Fertilisation Minérale : la Fertilisation Minérale Recommandée (FMR) et la demidose $(1 / 2 \mathrm{FMR})$. Les deux cultures maïs et sorgho en rotations à Koulikoro, le cotonnier et le maïs à Sikasso ont été cultivées chaque année sur des sous-parcelles alternées. Le sorgho et le maïs ont été semés à un écartement de $0,80 \times 0,40 \mathrm{~m}$, le cotonnier à $0,80 \times 0,30 \mathrm{~m}$ et $G$. sepium à 4,80 $\times 1 \mathrm{~m}$. L'année précédente le début de l'expérience, une homogénéisation des parcelles a été faite avec le niébé à Koulikoro et l'arachide à Sikasso. G. sepium a été installé pendant la saison de culture de juin 2016. L'année suivante, les plants de G. sepium bien établis ont été taillés à $0,5 \mathrm{~m}$ au-dessus du sol une à deux semaines avant la date prévue pour la plantation des cultures annuelles. Toute la biomasse de la parcelle individuelle a été incorporée à la zone de culture respective de chaque parcelle. Les arbres ont été à nouveau taillés lorsque le cotonnier, le maïs et le sorgho ont atteint une hauteur d'environ $60 \mathrm{~cm}$ et la biomasse a été appliquée entre les rangs des cultures. Les arbres étaient également taillés lorsqu'ils commençaient à ombrager les cultures et la biomasse des arbustes était appliquée au sol sous forme de paillis.

\section{Échantillonnage du sol et analyse physico- chimique}

Les échantillons de sol ont été prélevés après la récolte des cultures sous la forme d'un échantillon composite de cinq prises de sol prélevées à l'aide d'une tarière à une profondeur de 0 à $20 \mathrm{~cm}$. Les échantillons ont été homogénéisés avec un tamis de $2 \mathrm{~mm}$ et séchés 
à l'air à une température ambiante. Toutes les analyses de sol ont été effectuées au laboratoire de sol de l'Institut de recherche de l'agriculture biologique (FiBL) en Suisse. Toutes les méthodes ont été effectuées selon les méthodes de référence des stations de recherche agricole suisses (Agroscope, 1996-2018). La capacité maximale de rétention de l'eau (CMRE) a été déterminée après saturation capillaire en eau et sursaturation ultérieure d'un échantillon de sol. Le $\mathrm{pH}$ du sol a été déterminé avec une électrode dans une suspension d'eau du sol (1:1). La teneur en carbone organique total (COT) et en azote total (NT) des échantillons de sol a été déterminée à l'aide d'un analyseur élémentaire Variomax CNS (Elementar GmbH, Hanau,
Allemagne) en utilisant la méthode Dumas (Hansen, 1989). La teneur en azote minéral (Nmin) a été déterminée par spectroscopie sur l'analyseur Skalar 1.

\section{Analyse des données}

Le rendement et la composante du rendement ainsi que les données sur le sol ont été obtenus et analysés statistiquement à l'aide de l'analyse de variance. L'analyse de la variance a été réalisée à l'aide du logiciel JMP Pro 14 et R 3.5.1. Lorsque les valeurs F étaient significatives, la comparaison des moyennes entre les traitements a été faite avec le test HSD de Tukey.

Tableau 1 : Caractéristiques des deux sites d'étude.

\begin{tabular}{lll}
\hline Caractéristiques & Katibougou & Farako \\
\hline Zones agro-écologiques & Soudanienne & Soudano-Guinéenne \\
Région administrative & Koulikoro & Sikasso \\
Lieu & $12^{\circ} 57^{\prime} \mathrm{S}, 7^{\circ} 36^{\prime} \mathrm{E}$ & $11^{\circ} 33^{\prime} \mathrm{S}, 05^{\circ} 38^{\prime} \mathrm{E}$ \\
$\begin{array}{l}\text { Précipitations moyennes } \\
\text { annuelles }\end{array}$ & $600-800 \mathrm{~mm}$ & $800-1000 \mathrm{~mm}$ \\
Sols (Groupe de travail de & Tropical ferruginous (Ultisols) & Tropical ferruginous (Alfisols) \\
l'IUSS WRB, 2015) & & \\
& $21-26^{\circ} \mathrm{C}$ & $10-25^{\circ} \mathrm{C}$ \\
Végétation & Daniellia oliveri & Guiera senegalensis \\
(Sacande et al., 2016) & Vitellaria paradoxa & Parkia biglobosa \\
& Andropogon gayanus & Vitellaria paradoxa \\
& Andropogon pseudapricus & Terminalia laxiflora \\
& Terminalia laxiflora & Annona senegalensis \\
& Detarium microcarpum & Faidherbia albida \\
& Annona senegalensis & Daniellia oliveri \\
& Guiera senegalensis & Detarium microcarpum \\
& & Guiera senegalensis \\
& & Andropogon pseudapricus \\
& 0.25 < IAC < 0.50 & $0.25<$ IAC < 0.75 \\
index Aridité Climatique & Mil (Pennisetum americanum & Maïs (Zea mays L.) \\
Main crops & L Leeke) & Mil (Pennisetum americanum $\mathrm{L}$ \\
& Sorgh0 (Sorghum bicolor L.) & Leeke) \\
& Maïs (Zea mays L.) & Sorgho (Sorghum bicolor L.) \\
& & Coton (Gossypium hirsutum $\mathrm{L}$ ) \\
\hline
\end{tabular}




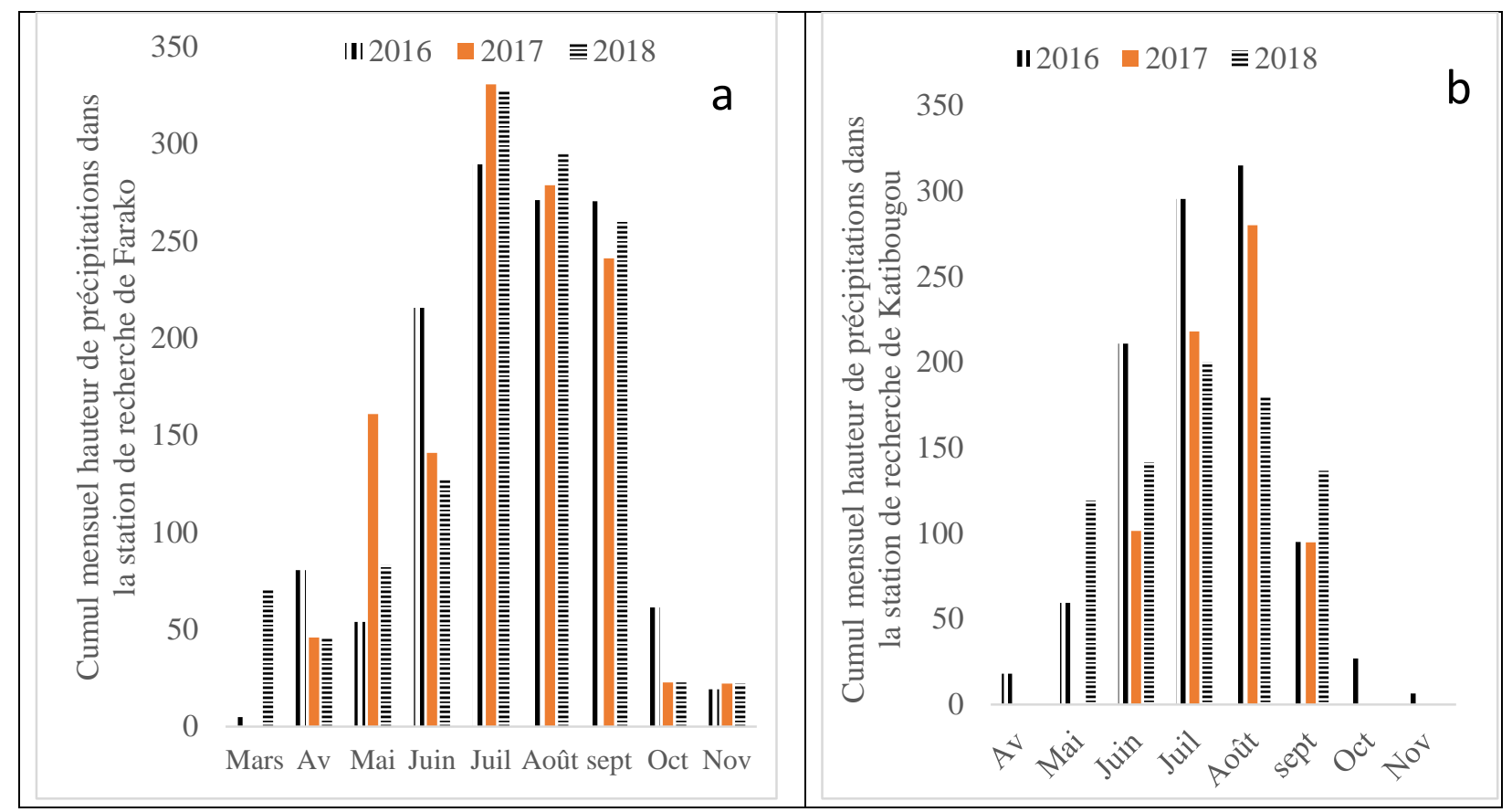

Figure 1 : Répartition mensuelle des précipitations dans les stations de recherche agronomique de Farako (a) et à l'IPR/IFRA de Katibougou (b).

Tableau 2 : Caractéristiques physico-chimiques des sols de référence à Sikasso et Koulikoro.

\begin{tabular}{llll}
\hline Variables évaluées & Farako & Katibougou \\
\hline Propriétés & Clay [\%] & 15 & 14 \\
physiques (\%) & Silt [\%] & 8.3 & 38.8 \\
& Sand [\%] & 76.5 & 47 \\
& Capacité Maximale de & & \\
& Rétention de l'eau [\%] & 25.3 & 27. \\
& Classe Texturale (WRB) & Sableux & Limoneux sableux \\
\hline Caractéristiques & $\mathrm{pH}\left(\mathrm{H}_{2} \mathrm{O}\right)$ & 5.4 & 5.4 \\
chimiques & $\mathrm{pH}(\mathrm{KCl})$ & 5.1 & 4.5 \\
& $\mathrm{Matière} \mathrm{organique} \mathrm{du} \mathrm{sol}$ & 0.11 & 0.17 \\
& {$[\%]$} & 0.01 & 0.03 \\
& $\mathrm{~N}[\%]$ & 1.32 & 3.8 \\
& $\mathrm{P}(\mathrm{ppm})$ & 4.17 & 7.54 \\
\hline
\end{tabular}


Tableau 3 : Les éléments nutritifs tels qu'appliqués avec des engrais minéraux et organiques sur les parcelles de terrain fertilisées à la dose recommandée.

\begin{tabular}{llccccc}
\hline & & $\mathbf{N}$ & $\mathbf{P}$ & $\mathbf{K}$ & $\mathbf{S}$ & $\mathbf{B}$ \\
\cline { 3 - 7 } Culture & Dose et type d'engrais & & & $\mathbf{k g ~ h a}^{-1}$ & & \\
\hline Maïs & $100 \mathrm{~kg} \mathrm{ha}^{-1} 15 \mathrm{~N}-15 \mathrm{P}-15 \mathrm{~K}$ & 15 & 15 & 15 & & \\
& $150 \mathrm{~kg} \mathrm{ha}^{-1}$ urée $46 \mathrm{~N}$ & 69 & & & & \\
& $5000 \mathrm{~kg} \mathrm{ha}^{-1}$ compost & 49 & 9 & 64 & & \\
\hline Coton & $200 \mathrm{~kg} \mathrm{ha}^{-1} 14 \mathrm{~N}-10 \mathrm{P}-10 \mathrm{~K}-7 \mathrm{~S}-1 \mathrm{~B}$ & 28 & 20 & 20 & 14 & \\
& $50 \mathrm{~kg} \mathrm{ha}^{-1}$ urée $46 \mathrm{~N}$ & 23 & & & & \\
& $5000 \mathrm{~kg} \mathrm{ha}^{-1}$ compost & 49 & 9 & 64 & & \\
\hline Sorgho & $100 \mathrm{~kg} \mathrm{ha}^{-1} 15 \mathrm{~N}-15 \mathrm{P}-15 \mathrm{~K}$ & 15 & 15 & 15 & & \\
& $50 \mathrm{~kg} \mathrm{ha}^{-1}$ urée $46 \mathrm{~N}$ & 23 & & & & \\
& $5000 \mathrm{~kg} \mathrm{ha}^{-1}$ compost & 49 & 9 & 64 & & \\
\hline
\end{tabular}

Tableau 4 : Biomasse issue de la taille du G. sepium et des apports de nutriments associés sur la base de la teneur moyenne en nutriments du G. sepium selon Kang (1984) $(4,21 \%$ N, 0,29\% P, 3,43\% K et $1,40 \mathrm{Ca}$ ).

\begin{tabular}{lcccccc}
\hline Site & Année & G. sepium (d.m.) & $\mathbf{N}$ & $\mathbf{P}$ & $\mathbf{K}$ & Ca \\
\cline { 4 - 7 } & & & \multicolumn{3}{c}{$\left[\mathbf{k g ~ h a}^{-\mathbf{1}} \mathbf{y r}^{-\mathbf{1}}\right]$} \\
Farako & 2017 & 3500 & 147,35 & 10,15 & 120,05 & 49 \\
\multirow{3}{*}{ Katibougou } & 2018 & 3600 & 151,56 & 10,44 & 123,48 & 50,4 \\
& 2017 & 1400 & 58,94 & 4,06 & 48,02 & 19,6 \\
& 2018 & 1600 & 67,36 & 4,64 & 54,88 & 22,4 \\
\hline Zoumana Diassa & 2017 & 2800 & 117,88 & 8,12 & 96,04 & 39,2 \\
\multirow{3}{*}{ Mafèya } & 2018 & 2500 & 105,25 & 7,25 & 85,75 & 35 \\
& 2017 & 1200 & 50,52 & 3,48 & 41,16 & 16,8 \\
& 2018 & 1400 & 58,94 & 4,06 & 48,02 & 19,6 \\
\hline
\end{tabular}

\section{RESULTATS}

Le maïs et le cotonnier ont été cultivés dans la région de Sikasso dans le cadre des essais en station et en milieu paysan. Dans la région de Koulikoro, le maïs et le sorgho ont été cultivés. Les deux cultures étaient cultivées chaque année en rotation entre les deux sousparcelles. Lors des essais en station, le rendement en grains de maïs en 2017 était de 43\% plus élevé à Farako qu'à Katibougou et de $100 \%$ plus élevé en 2018. Dans les exploitations agricoles, le rendement du maïs à Zoumana-Diassa en 2017 était supérieur de 63\%, alors qu'en 2018 il était inférieur de 36\% à celui de Maféya.

Maïs et sorgho dans la région de Koulikoro

A l'IPR/IFRA de Katibougou, l'analyse de variance a montré pour le rendement de la culture du maïs une différence non significative $(p=0,198)$ en 2017 et significative $(p=0,015)$ en 2018 entre les systèmes 
agroforestiers avec Gliricidia sepium et sans Gliricidia sepium. La biomasse du Gliricidia sepium utilisée pour le mulch a permis d'augmenter les rendements du maïs de $28 \%$ en 2017 et de $68 \%$ en 2018. Pour la culture du sorgho, des différences significatives ont été observées en 2017 ( $\mathrm{p}=0,032)$ et en 2018 ( $\mathrm{p}=$ 0,003 ) entre les deux systèmes culture pour le rendement. Les rendements ont augmenté de $35 \%$ en 2017 et de $104 \%$ en 2018 par rapport aux rendements observés sur les parcelles témoins (Tableau 5). Les essais en milieu paysan à Mafèya ont montré une tendance de rendement plus élevé avec G. sepium et son paillis. La culture du maïs a présenté une différence significative $(p=0,0004)$ entre des niveaux de fertilisation en 2017 et non significative $(\mathrm{p}=0,541)$ en 2018 pour le rendement (Tableau 6).

En 2018, les rendements des cultures ont été beaucoup plus élevés qu'en 2017, même avec la demi-dose de fertilisation minérale. Par conséquent, en général les conditions de croissance ont probablement été bien meilleures. L'amendement d'engrais organique n'a rattrapé les autres traitements qu'en 2018 avec la culture du maïs, où tous les traitements semblaient donner de bons rendements grâce à des pluies suffisantes. Par rapport aux essais en milieu paysan, les rendements du maïs et du sorgho dans la station en 2017 ne différaient pas beaucoup. En 2018, les rendements du sorgho et du maïs étaient respectivement 139 et $79 \%$ plus élevés en milieu paysan qu'en station dans la région de Koulikoro.

\section{Le maïs et le cotonnier dans la région de Sikasso}

Dans des essais en station à Farako, le rendement en fibres des graines de cotonnier, des différences significatives $(\mathrm{p}=0,016)$ en 2017 et $(\mathrm{p}=0,002)$ en 2018 ont été observées entre les deux systèmes de culture. La biomasse du Gliricidia sepium utilisée comme mulch a permis d'augmenter les rendements du cotonnier à Farako de $27 \%$ en 2017 et $34 \%$ en 2018 par rapport aux rendements observés dans les parcelles témoins sans mulch. (Tableau 7). A la sous-station de Farako, l'analyse de variance pour le rendement la culture du maïs n'a pas permis de montrer une différence non significative $(p=0,158)$ en 2017. En 2018, la différence a été significative $(\mathrm{p}=0,047)$ entre les systèmes agroforestiers avec Gliricidia sepium et sans Gliricidia sepium. Dans les mêmes conditions, la production de graines a été améliorée de $19 \%$ en 2017 et $58 \%$ en 2018 par rapport au témoin (Tableau 7). À la demidose, le rendement a diminué de 15\% en 2017 et de $31 \%$ en 2018 . Avec l'engrais organique, le rendement était de 60 à $70 \%$ inférieur à celui du système avec engrais minéral à la dose recommandée.

Dans les essais en milieu paysan à Zoumana Diassa, pour la culture du cotonnier, l'analyse de variance a montré une différence significative ( $p=0,0001)$ entre les traitements pour le rendement en 2017. En 2018, il n'y a pas eu de différence significative entre les traitements $(\mathrm{p}=0,548)$ pour le rendement (Tableau 8). En 2017, les rendements en milieu paysan étaient environ $50 \%$ plus élevés qu'à la station. En 2018, il n'y avait pas beaucoup de différence entre les résultats des rendements en milieux paysans et ceux de la station.

\section{Effet des applications de paillis de G. sepium sur la qualité des sols en 2018 après deux saisons de culture \\ Le $\mathrm{pH}$ de Farako était légèrement} inférieur à celui de Katibougou. L'effet de l'introduction de la biomasse de G. sepium a augmenté la valeur du pH (Tableau 9), tandis que les types et les quantités d'engrais n'ont eu aucun effet sur le $\mathrm{pH}$.

Dans les sites, il n'y a une différence hautement significative pour le stock de COS $(\mathrm{p}=0,0001)$. Pour les systèmes de culture il $\mathrm{y}$ $\mathrm{a}$ une différence significative avec $(\mathrm{p}=$ 0,0183). Pour l'azote totale, dans les sites, il n'y a une différence hautement significative pour le stock de COS ( $p<0,0001)$. Pour les systèmes de culture il y a une différence significative avec $(p=0,0049)$. Pour la fertilisation, il n'y a pas eu de différence significative entre les traitements pour le stock de $\operatorname{COS}(\mathrm{p}=0,3253)$. Sur les deux sites, la teneur en carbone organique (Corg) du sol et en azote total $(\mathrm{TN})$ était inférieure à $1 \%$ (Tableau 10). En 2018, la teneur en Corg à Farako, avec 
la texture sableuse du sol, était en moyenne de 2,1 $\mathrm{mg} \mathrm{kg}^{-1}$ et à Katibougou, de $3,7 \mathrm{mg} \mathrm{kg}^{-1}$, soit $80 \%$ de plus qu'à Farako, avec un sol à texture limono-sableuse. La teneur en azote totale à Farako était de $0,2 \mathrm{mg} \mathrm{kg}^{-1}$ et à Katibougou 0,3 $\mathrm{mg} \mathrm{kg}^{-1}$, soit une augmentation de $50 \%$ par rapport à Farako. Les rapports $\mathrm{C} / \mathrm{N}$ étaient donc légèrement plus élevés à Katibougou $(12,1)$ qu'à Farako $(10,3)$.

$\mathrm{Au}$ cours de la troisième année après la plantation de $G$. sepium et deux saisons de culture, le carbone organique et l'azote totale du sol étaient de 5 à $6 \%$ plus élevés dans les parcelles avec G. sepium que dans celles sans G. sepium (Tableau 10). L'effet des engrais minéraux et organiques était relativement faible et non significatif. En comparant chaque amendement fertilisant avec et sans G. sepium, seuls l'engrais organique à demi-dose et le témoin non fertilisé à Katibougou ont montré des valeurs de Corg et de TN avec G. sepium inférieures à celles des témoins sans G. sepium. Tous les autres ont montré une augmentation de cet important indicateur de la qualité du sol.

Tableau 5 : Rendement des grains de sorgho et de maïs en station à l'IPR/IFRA de Katibougou, en 2017 et 2018.

\begin{tabular}{|c|c|c|c|c|c|c|c|}
\hline \multirow{2}{*}{\multicolumn{2}{|c|}{$\begin{array}{l}\text { Système de culture } \\
\text { Traitement fertilisation }\end{array}$}} & \multicolumn{2}{|c|}{ Rdt maïs grain [kg ha-1] } & \multicolumn{4}{|c|}{ Rdt sorgho grain [kg ha-1] } \\
\hline & & 2017 & 2018 & \multicolumn{2}{|c|}{2017} & \multicolumn{2}{|c|}{2018} \\
\hline$+G$. sepium & FMR & 1932 a & $1385 \mathrm{ab}$ & 1448 & $\mathrm{a}$ & 2674 & $\mathrm{a}$ \\
\hline$+G$. sepium & $1 / 2 \mathrm{FMR}$ & $1271 \mathrm{abc}$ & 1439 a & 1125 & $a b$ & 2154 & $a b$ \\
\hline$+G$. sepium & FOR & $761 \mathrm{~cd}$ & $1065 \mathrm{bc}$ & 1188 & $a b$ & 1356 & bcd \\
\hline$+G$. sepium & $1 / 2 \mathrm{FOR}$ & $401 \mathrm{~d}$ & $1200 \mathrm{abc}$ & 698 & $\mathrm{bc}$ & 1381 & bcd \\
\hline$+G$ sepium & SFM & $360 \mathrm{~d}$ & $975 \mathrm{~cd}$ & 771 & $\mathrm{bc}$ & 975 & cde \\
\hline -G. sepium & FMR & $1500 \mathrm{ab}$ & 860 cde & 1057 & abc & 1903 & $a b c$ \\
\hline -G. sepium & $1 / 2 \mathrm{FMR}$ & $1193 \mathrm{bc}$ & $972 \mathrm{~cd}$ & 776 & $\mathrm{bc}$ & 1621 & $a b c$ \\
\hline -G. sepium & FOR & $328 \mathrm{~d}$ & 556 e & 839 & $\mathrm{bc}$ & 291 & de \\
\hline -G. sepium & $1 / 2 \mathrm{FOR}$ & $448 \mathrm{~d}$ & 570 e & 667 & $\mathrm{bc}$ & 221 & de \\
\hline -G. sepium & SFM & $219 \mathrm{~d}$ & $650 \mathrm{de}$ & 542 & $\mathrm{c}$ & 157 & $\mathrm{e}$ \\
\hline \multicolumn{8}{|l|}{ ANOVA } \\
\hline \multicolumn{8}{|l|}{ Effets } \\
\hline G. sepium & & $128 \%$ & $168 \% *$ & $135 \% *$ & \multicolumn{3}{|c|}{$204 \% *$} \\
\hline Fertilisation & & $*$ & & & & * & \\
\hline
\end{tabular}

Tableau 6 : Rendement des grains de sorgho et de maïs à Mafèya, région de Koulikoro en 2017 et 2018.

\begin{tabular}{|c|c|c|c|c|c|c|c|c|}
\hline \multirow[b]{3}{*}{ Système de culture } & \multicolumn{4}{|c|}{ Sorgho } & \multicolumn{4}{|c|}{ Maïs } \\
\hline & \multicolumn{2}{|c|}{2017} & \multicolumn{2}{|c|}{2018} & \multicolumn{2}{|c|}{2017} & \multicolumn{2}{|c|}{2018} \\
\hline & & & & $\mathrm{kg} \mathrm{l}$ & & & & \\
\hline FMR $+G$. sepium mulch & 1096 & $\mathrm{a}$ & 3486 & $\mathrm{a}$ & 1431 & a & 3978 & $\mathrm{a}$ \\
\hline FMR & 961 & $a b$ & 2610 & $a b$ & 997 & $a b$ & 2925 & $\mathrm{a}$ \\
\hline $1 / 2 \mathrm{FMR}+$ G.sepium mulch & 734 & $\mathrm{~b}$ & 1880 & $\mathrm{~b}$ & 823 & $\mathrm{~b}$ & 2203 & a \\
\hline $1 / 2 \mathrm{FMR}+\mathrm{FOR}$ & 808 & $a b$ & 1252 & $\mathrm{~b}$ & 1141 & $a b$ & 2936 & $\mathrm{a}$ \\
\hline
\end{tabular}


Tableau 7 : Rendement des grains de maïs et en fibres de cotonnier dans la sous station de Farako, IER/CRRA, région de Sikasso, en 2017 et 2018.

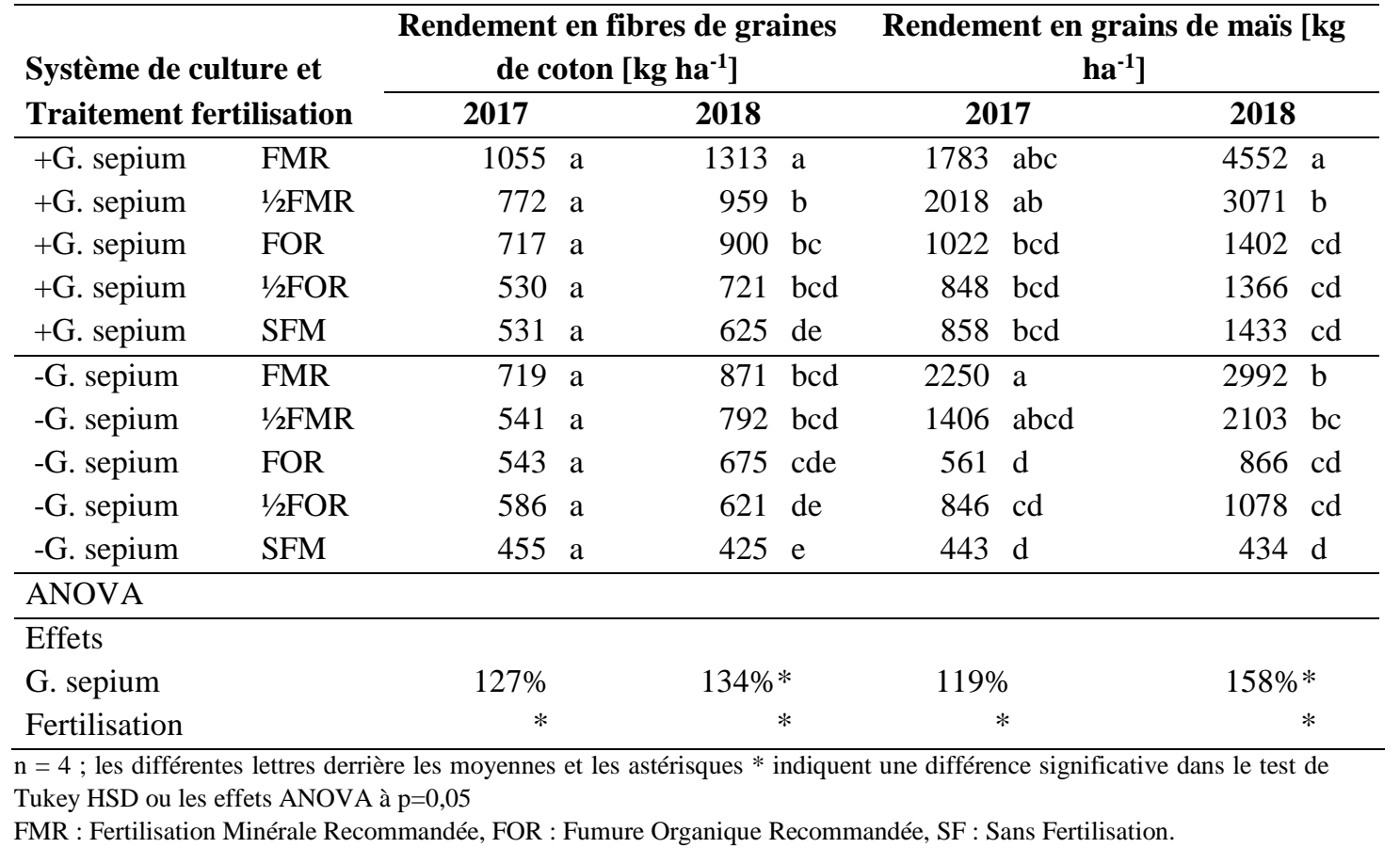

Table 8 : Rendement des grains de maïs et en fibres de cotonnier à Zoumana-Diassa, région de Sikasso, en 2017 et 2018.

\begin{tabular}{|c|c|c|c|c|c|c|c|c|}
\hline \multirow{3}{*}{ Traitement } & \multicolumn{4}{|c|}{ Maïs } & \multicolumn{4}{|c|}{ Cotonnier } \\
\hline & \multicolumn{2}{|c|}{2017} & 2018 & & \multicolumn{2}{|c|}{2017} & \multicolumn{2}{|r|}{2018} \\
\hline & \multicolumn{8}{|c|}{$\mathrm{kg} \mathrm{ha}^{-1}$} \\
\hline FMR + G. sepium mulch & 1945 & $\mathrm{a}$ & 2213 & $\mathrm{a}$ & 903 & $\mathrm{a}$ & 728 & a \\
\hline FMR & 1436 & a & 1959 & $\mathrm{a}$ & 1020 & $\mathrm{a}$ & 646 & $\mathrm{a}$ \\
\hline $1 / 2 \mathrm{FMR}+$ G. sepium mulch & 1869 & a & 1924 & a & 767 & $\mathrm{a}$ & 696 & $\mathrm{a}$ \\
\hline $1 / 2 \mathrm{FMR}+\mathrm{FOR}$ & 1918 & $\mathrm{a}$ & 1631 & $\mathrm{a}$ & 793 & $\mathrm{a}$ & 472 & $\mathrm{a}$ \\
\hline
\end{tabular}

$\mathrm{n}=15$; les différentes lettres derrière les moyennes indiquent une différence significative dans le test de Tukey HSD à $\mathrm{p}=0,05$

FMR : Fertilisation Minérale Recommandée, FOR : Fumure Organique Recommandée.

Tableau 9 : Effets du système de culture sur le pH du sol (eau) Sikasso et Koulikoro

\begin{tabular}{|c|c|c|c|c|c|}
\hline $\begin{array}{l}\text { Système de culture et } \\
\text { Traitement fertilisation }\end{array}$ & & $\begin{array}{l}\text { Farako } \\
\text { pH }(\text { H2O })\end{array}$ & SD & $\begin{array}{l}\text { Katibougou } \\
\text { pH (H2O) }\end{array}$ & SD \\
\hline+ G. sepium & FMR & 6.4 & 0.4 & 6.701 & 0.399 \\
\hline +G. sepium & $1 / 2 \mathrm{FMR}$ & 6.5 & 0.4 & 6.699 & 0.347 \\
\hline +G. sepium & FOR & 6.6 & 0.4 & 6.763 & 0.330 \\
\hline+ G. sepium & $1 / 2 \mathrm{FOR}$ & 6.4 & 0.4 & 6.585 & 0.435 \\
\hline +G. sepium & SFM & 6.5 & 0.4 & 6.670 & 0.334 \\
\hline -G. sepium & FMR & 6.0 & 0.6 & 6.180 & 0.593 \\
\hline -G. sepium & $1 / 2 \mathrm{FMR}$ & 6.0 & 0.6 & 6.181 & 0.541 \\
\hline -G. sepium & FOR & 6.2 & 0.5 & 6.420 & 0.411 \\
\hline
\end{tabular}




\begin{tabular}{|c|c|c|c|c|c|}
\hline -G. sepium & $1 / 2 \mathrm{FOR}$ & 6.1 & 0.7 & 6.351 & 0.503 \\
\hline -G. sepium & SFM & 6.1 & 0.6 & 6.434 & 0.394 \\
\hline \multicolumn{6}{|l|}{ ANOVA } \\
\hline \multirow[t]{2}{*}{ Sites } & Farako & 6.3 & $\mathrm{~b}$ & & \\
\hline & Katibougou & 6.5 & $\mathrm{a}$ & & \\
\hline \multirow[t]{2}{*}{ Système de culture } & + G. sepium & 6.6 & $\mathrm{a}$ & & \\
\hline & -G. sepium & 6.2 & $\mathrm{~b}$ & & \\
\hline \multirow[t]{3}{*}{ Effets Facteur } & Site & 0.0031 & & & \\
\hline & $\begin{array}{l}\text { Système de } \\
\text { culture }\end{array}$ & $<.0001$ & & & \\
\hline & Fertilisation & 0.5529 & & & \\
\hline
\end{tabular}

FMR : Fertilisation Minérale Recommandée, FOR : Fumure Organique Recommandée, SF : Sans Fertilisation. $(n=40)$

Tableau 10 : Effets des systèmes culturaux et de l'épandage d'engrais sur le carbone organique du sol et l'azote total en station à Farako et à Katibougou en 2018.

\begin{tabular}{|c|c|c|c|c|c|c|c|c|c|}
\hline \multirow{2}{*}{\multicolumn{2}{|c|}{$\begin{array}{l}\text { Système de culture et } \\
\text { Traitement fertilisation }\end{array}$}} & \multicolumn{2}{|c|}{ Farako } & \multicolumn{2}{|c|}{ Katibougou } & \multicolumn{2}{|c|}{ Farako } & \multicolumn{2}{|c|}{ Katibougou } \\
\hline & & \multirow{2}{*}{$\begin{array}{c}\text { Corg } \\
{\left[\mathrm{mg} \mathrm{kg}^{-1}\right]}\end{array}$} & \multirow{2}{*}{$\begin{array}{l}\text { SD } \\
0.37\end{array}$} & \multirow{2}{*}{$\begin{array}{c}\text { Corg } \\
{\left[\begin{array}{c}\left.\mathbf{m g ~ k g}^{-1}\right] \\
4.07\end{array}\right.}\end{array}$} & \multirow{2}{*}{$\begin{array}{l}\text { SD } \\
0.51\end{array}$} & \multirow{2}{*}{$\begin{array}{c}\begin{array}{c}\mathbf{N t} \\
{\left[\mathrm{mg} \mathrm{kg}^{-1}\right]}\end{array} \\
0.215\end{array}$} & \multirow{2}{*}{$\begin{array}{r}\text { SD } \\
0.031\end{array}$} & $\begin{array}{l}\mathrm{Nt} \\
\left.\mathrm{kg}^{-1}\right]\end{array}$ & \multirow{2}{*}{$\begin{array}{l}\text { SD } \\
0.038\end{array}$} \\
\hline+ G. sepium & FMR & & & & & & & 0.349 & \\
\hline +G. sepium & $1 / 2 \mathrm{FMR}$ & 2.13 & 0.21 & 3.90 & 0.74 & 0.204 & 0.031 & 0.320 & 0.055 \\
\hline$+G$. sepium & FOR & 2.09 & 0.24 & 3.66 & 0.47 & 0.206 & 0.028 & 0.301 & 0.041 \\
\hline +G. sepium & $1 / 2 \mathrm{FOR}$ & 2.16 & 0.38 & 3.46 & 0.78 & 0.213 & 0.037 & 0.291 & 0.062 \\
\hline$+G$. sepium & SFM & 2.14 & 0.28 & 3.47 & 0.46 & 0.204 & 0.029 & 0.284 & 0.03 \\
\hline -G. sepium & FMR & 1.95 & 0.41 & 3.57 & 0.66 & 0.186 & 0.031 & 0.286 & 0.04 \\
\hline -G. sepium & $1 / 2 \mathrm{FMR}$ & 1.84 & 0.29 & 3.62 & 0.46 & 0.188 & 0.028 & 0.315 & 0.033 \\
\hline -G. sepium & FOR & 2.06 & 0.32 & 3.36 & 0.42 & 0.203 & 0.03 & 0.279 & 0.031 \\
\hline -G. sepium & $1 / 2 \mathrm{FOR}$ & 2.01 & 0.29 & 3.70 & 0.38 & 0.198 & 0.029 & 0.296 & 0.034 \\
\hline -G. sepium & SFM & 1.94 & 0.25 & 3.74 & 0.64 & 0.188 & 0.023 & 0.305 & 0.039 \\
\hline
\end{tabular}

ANOVA

résultats

\begin{tabular}{lccccc}
\hline Sites & Farako & 2.05 & $\mathrm{~b}$ & 0.200 & $\mathrm{~b}$ \\
& Katibougou & 3.65 & $\mathrm{a}$ & 0.303 & $\mathrm{a}$ \\
\hline $\begin{array}{l}\text { Système de } \\
\text { culture }\end{array}$ & + G. sepium & 2.93 & $\mathrm{a}$ & 0.259 & $\mathrm{a}$ \\
& & & & & \\
& -G. sepium & 2.78 & $\mathrm{~b}$ & 0.244 & $\mathrm{~b}$ \\
\hline Effets Facteurs & Site & $<.0001$ & & $<.0001$ & \\
& Système de culture & 0.0183 & & 0.0049 \\
& $\quad$ Fertilisation & 0.5075 & & 0.3253
\end{tabular}

NB : FMR : Fertilisation Minérale Recommandée, FOR : Fumure Organique Recommandée, SF : Sans Fertilisation. 


\section{DISCUSSION}

\section{Production de biomasse de G. sepium}

Les quantités annuelles moyennes de production de biomasse (matière sèche) de $G$. sepium étaient de 3,5 t.ha ${ }^{-1}$ à Farako dans la zone soudano-guinéenne et de 1,5 t.ha- ${ }^{-1}$ à Katibougou dans la zone soudanaise. La biomasse issue de l'élagage dans les exploitations était en moyenne de 2,6 t.ha ${ }^{-1}$ à Zoumana Diassa et de 1,3 t.ha ${ }^{-1}$ à Mafèya. Ces chiffres se situent dans la fourchette de 2,5 à 5,7 t.ha $^{-1}$ pour Farako et en dessous pour Katibougou (Vanlauwe et al., 2005). En supposant une teneur en biomasse $\mathrm{N}$ d'environ $4 \%$ et aucun apport d'azote inorganique, $G$. Sepium doit produire au moins 4 t.ha $^{-1}$ de matière sèche pour répondre aux besoins en azote du maïs et du cotonnier. Dans la présente étude, la quantité maximale était de 3,5 t.ha ${ }^{-1}$ à Farako. Une production de biomasse plus faible de G. Sepium dans des conditions plus sèches a également été signalée par Okonkwo et al. (2009). Il apparaît que la production de de 1,5 t.ha ${ }^{-1}$ biomasse dans le site de Katibougou qui est sec, n'est pas suffisante pour répondre à la demande en $\mathrm{N}$ des cultures et doit être amendée avec des engrais inorganiques pour une production optimale des cultures.

\section{Propriétés physiques et chimiques des sols}

Les systèmes de culture en couloire avec G. sepium et l'application de sa biomasse comme paillis produit à partir de celui-ci peuvent aider à maintenir et à améliorer les caractéristiques physiques et chimiques des sols (Smethurst et al., 2017, Diouf et al., 2008 ;; Kuyah et al., 2019). Les avantages des systèmes de culture en couloir dans l'agriculture des écosystèmes arides sont si importants qu'il est surprenant de constater que le faible taux d'adoption et de développement de ce système agricole justifie une étude plus approfondie (Luedeling et al., 2016). Le recours aux pratiques de l'agroforesterie pour les cultures annuelles présente des avantages en ce qui concerne la disponibilité de l'azote, la disponibilité de l'eau, la biomasse à utiliser comme fourrage, amendement du sol, les barrières vivantes pour arrêter l'érosion du sol et de nouvelles possibilités d'emploi. Ce n'est qu'en ce qui concerne le rendement des cultures et la qualité des sols, mesurés en $\mathrm{pH}$, en carbone organique et en azote organique, que les effets du système de culture en couloir avec G. sepium étaient déjà significatifs après trois ans d'étude. Compte tenu de la pauvreté des sols sur les sites étudiés, l'augmentation de la teneur en carbone organique constitue une amélioration précieuse de la qualité des sols. Non seulement elle contribue à la séquestration du $\mathrm{CO} 2$ dans les sols, mais elle peut aussi améliorer la structure des sols, leur capacité de rétention d'eau, leur porosité et leur fertilité (Baveye et al., 2016 ; FAO, 2017 ; Van der Wal et de Boer, 2017). Dans les écosystèmes arides, le rôle et les avantages de la matière organique du sol sont particulièrement liés aux propriétés physiques, biologiques et chimiques des sols (McBratney et al., 2014). Les résultats obtenus soutiennent également l'idée que l'amélioration de la qualité des sols ainsi que d'autres conditions de croissance des cultures rendent les sols et le système agricole sensibles aux engrais, ce qui est l'un des objectifs de la gestion intégrée de la fertilité des sols (GIFS) (Vanlauwe et al., 2011 ; Fairhurst, 2015).

Les niveaux d'azote total ont augmenté en même temps que la teneur en matière organique et ont montré des avantages pour les systèmes avec G. sepium. Son paillis a un faible rapport $\mathrm{C} / \mathrm{N} \quad(<12)$, est facilement décomposable (Adekiya, 2018 ; Akintan, 2019) et sert de nutriment végétal facilement disponible fournissant de la matière organique qui peut entrer dans le bassin de carbone organique $\mathrm{du}$ sol. Les résultats obtenus confirment l'efficacité accrue de l'utilisation d'engrais, lorsqu'une culture permanente telle que G. sepium protège le champ. Rendement des grains de cotonnier, du maïs et du sorgho

L'introduction de G. sepium a amélioré les rendements du cotonnier, du maïs et du sorgho, ce qui soutient les tentatives de promotion de l'agroforesterie et des systèmes de culture en couloir en Afrique de l'Ouest. $G$. sepium affecte la quantité et la qualité des composants chimiques, biologiques et physiques du sol, ce qui améliore les rendements. Les résultats des essais de cultures intercalaires dans des stations de recherche au 
Mali confirment le potentiel de G. sepium pour améliorer la fertilité des sols et les rendements des cultures associées ( Kouadio et al 2014).

Le rendement du maïs s'est amélioré de $19 \%$ en 2017 et de $56 \%$ en 2018 par rapport au contrôle sans G. sepium à Farako. A Katibougou, une amélioration de $28 \%$ en 2017 et de $61 \%$ en 2018 a été observée par rapport au contrôle sans $G$. sepium. Le rendement en grains de sorgho s'est amélioré de 35\% en 2017 et de 34\% en 2018 par rapport au contrôle sans G. sepium. Ces résultats confirment ceux obtenus au Sénégal par Diouf et al. (2008), qui ont montré que l'utilisation du paillis de $G$. sepium comme engrais vert augmente la biomasse de maïs et des rendements céréaliers de $25 \%$ et $9 \%$ respectivement par rapport au contrôle sans paillis de G. sepium. Selon la même source, lorsque la biomasse utilisée provient de plants de G. sepium inoculés avec des souches de Rhizobium et de champignons mycorhiziens en combinaison, le rendement en grains du maïs augmente de $130 \%$ par rapport au contrôle.

Les résultats des essais de culture en couloir avec Gliricidia sepium confirment le potentiel d'amélioration des rendements en maïs et en sorgho. Rosenstock et al. (2014) ont démontré l'état du taux possible de fixation de G. sepium à l'azote de $170-204 \mathrm{~kg} \mathrm{~N}^{-} \mathrm{ha}^{-1}$, ce qui est un énorme capital que ces arbustes en culture intercalaire fournissent. Dans les deux stations (IPR/IFRA de Katibougou et Farako) et en milieu rural, les rendements de maïs ont varié entre 1134 et $4500 \mathrm{~kg} \cdot \mathrm{ha}^{-1}$. Ces résultats sont proches de ceux obtenus en Afrique australe où l'utilisation de la litière de Gliricidia sepium a maintenu les rendements de maïs entre 1800 et 3000 kg.ha ${ }^{-1}$ (Mafongaya et al., 2003). Ces auteurs ont indiqué que le rendement dépend souvent de la qualité de la biomasse. La faible capacité de rétention d'eau (Tableau 2) fait que les systèmes de production dépendent d'une pluviométrie constante sur les deux sites.

\section{Conclusion}

A l'issue de ces travaux, il apparaît que G. sepium utilisé comme engrais vert ou en combinaison avec des engrais minéraux et du compost sur trois saisons de croissance a amélioré significativement le $\mathrm{pH}$ du sol, le carbone organique et l'azote total. Les rendements des cultures de cotonnier, de sorgho et de maïs ont été beaucoup plus élevés, doublant parfois en raison de l'association avec G. sepium. Les producteurs participants ont apprécié le système de culture en couloir avec G. sepium en raison des rendements plus élevés et de la facilité de la technologie. Par conséquent, les conclusions à tirer de cette étude est que la culture en couloir avec $G$. sepium est une technologie qui a un fort potentiel d'extensibilité pour les producteurs des zones soudaniennes et soudano-guinéennes du Mali, qui ont déjà exprimé leur intérêt lors des journées agricoles et développé des pépinières pour diffuser cette technique. Malgré les résultats intéressants obtenus, les producteurs expriment également des doutes, car les espèces recommandées pour le système de culture en couloir ne fournissent pas de (sous-produits) comestibles, ce qui semble décourager les producteurs à adopter cette technologie. Ils voient également des limites dans le manque de main-d'œuvre aux heures de pointe où l'entretien périodique des allées d'arbustes devrait avoir lieu et dans la nécessité de protéger les jeunes plantes qui ne sont pas encore complètement à l'abris des animaux.

\section{CONFLIT D'INTERESTS}

Les auteurs ne déclarent aucun conflit d'intérêts.

\section{CONTRIBUTIONS DES AUTEURS}

Conceptualisation : SD, SGD, FS, NA et AF; Analyse formelle : SD, $\mathrm{HC}$ et $\mathrm{AF}$; Investigation, SD, SGD, FS, NA, HC et AF; Administration de projet : FS ; Supervision : SGD, FS et AF; Validation : AF, NA, HC; Rédaction du projet original : $\mathrm{SD}$ et $\mathrm{AF}$; Révision et édition, SGD, FS, NA, HC et AF. Tous les auteurs ont lu et accepté la version publiée du manuscrit.

\section{REMERCIEMENTS}

Nous reconnaissons et remercions le Programme suisse de recherche sur les enjeux mondiaux du développement (programme 
r4d), lancé par la Direction du Développement et de la Coopération (DDC) et le Fonds National Suisse (FNS) d'avoir soutenu le projet "Farmer driven organic resource management to build soil fertility (ORM4Soil) au Mali et de nous avoir donné l'opportunité de mener cette recherche. (Subvention numéro : 400540152224. Pour plus de détails sur le projet, consultez le site www.orm4soil.net). Nous tenons à remercier Anton Kuhn et Adolphe Munyangabe pour leur assistance technique qualifiée dans le laboratoire de FiBL. Les auteurs remercient les producteurs participants, les techniciens de terrain et les nombreuses personnes qui ont apporté des appuis techniques. Merci également aux trois réviseurs pour leurs commentaires détaillés qui nous ont aidés à améliorer le manuscrit.

\section{RÉFÉRENCES}

Adekiya AO. 2018. Legume Mulch Materials and Poultry Manure Affect Soil Properties, and Growth and Fruit Yield of Tomato. Int. J. Agric. Conspec. Sci., 83(2): 161-167. DOI: $10.2478 /$ johr2018-0008

Agroscope. 1996-2018. Referenzmethoden der Eidgenössischen landwirtschaftlichen Forschungsanstalten (1e édn).

Bodenuntersuchung zur Düngeberatung: Zürich-Reckenholz.

Akintan CI. 2019. Agronomic Performance of Maize (Zea mays L.) as influenced by Leaf Pruning Quality in a Cut and Carry Agroforestry System in Akure (Ondo State, Nigeria). Int. J. KIU Humanities., 4(3): $\quad$ 199-208. DOI: http://www.ijhumas.com/ojs/index.php/k iuhums/article/view/627

Bado BV, Savadogo P, Manzo, MLS. 2016. Restoration of Degraded Lands in West Africa Sahel: Review of experiences in Burkina Faso and Niger. Technical Report. ICRISAT.

Baveye PC, Baveye J, Gowdy J. 2016. Soil "Ecosystem" Services and Natural Capital: Critical Appraisal of Research on Uncertain Ground. Int. J. Front. Environ. Sci., 41(4). DOI: https://doi.org/10.3389/fenvs.2016.0004 1

Bayala J, Sanou J, Teklehaimanot Z, Kalinganine A, Ouédraogo SJ. 2014. Parklands for buffering climate risk and sustaining agricultural production in the Sahel of West Africa. Int. J. Elsevier., 6: 28-34.

DOI: http://dx.doi.org/10.1016/j.cosust.2013.1 0.004

Beedy TL, Snapp SS, Akinnifesi FK, Sileshi GW. 2010. Impact of Gliricidia sepium Intercropping on soil organic matter fraction in a maize-based cropping system. Int. J. Agric. Ecosys. Environ., 138: 139-146. DOI: https://doi.org/10.1016/j.agee.2010.04.00 8

Cobbina J, Ata-Krah AN, Meregini AO, Duguma B. 1990. Productivity of some browse plants on soils of southeastern Nigeria. Tropical Grasslands, 24(1): 4145.

Compagnie Malienne de Développement des Textiles (CMDT). 2018. Annuaire statistique 2016/2017. Résultats de l'enquête agricole permanente. In: (DPCG), D.d.l.p.d.c.g. (éd.). CMDT : Bamako.

Dembele D, Traore K, Quansah C, Osei Jnr EM, Bocar DSBA, Ballo M. 2016. Optimizing Soil Fertility Management Decision in Mali by Remote Sensing and GIS. Int. J. Agricultural Research., 3(4): 022-034.

DOI: http://www.donnishjournals.org/dja

Diouf A, Diop T, Fall-Ndiaye M, Gueye M. 2008. Impact de la biomasse de Gliricidia sepium utilisée comme engrais vert sur la culture du mais (Zea mays) au Sénégal. Int. J. Sci., $\quad \mathbf{8}(3)$ : $\quad 10-17$. http://www.ucadjds.org

Direction Nationale de l'Agriculture (DNA). 2017. Rapport annuel 2017. In: l'Agriculture, M.d. (Ed.). Ministère de l'Agriculture : Bamako, Mali.

Fairhurst T. 2015. Manuel de Gestion Intégrée de la Fertilité des Sols (edn). Consortium Africain pour la Santé des Sols : Nairobi. 
FAO. 2017. Soil Organic Carbon: the Hidden Potential. Food and Agriculture Organization of the United Nations : Rome.

FAO, FIDA, PAM. 2015. L'Etat de l'insecurité alimentaire dans le monde. Objectifs internationaux 2015 de réduction de la faim: des progrès inégaux. FAO : Rome.

Hansen B. 1989. Determination of Nitrogen as Elementary N, an Alternative to Kjeldahl. Int J. Acta Agriculturae Scandinavica., 39: 113-118. DOI: https://doi.org/10.1080/00015128909438 504

USS Working Group WRB. 2015. World Reference Base for Soil Resources 2014, update 2015. International soil classification system for naming soils and creating legends for soil maps., World Soil Resources Reports. FAO : Rome.

Jabbar MA, Reynolds L, Larbi A, Smith J. 1997. Nutritional and economic benefits of Leucaena and Gliricidia as feed supplements for small ruminants in humid West Africa. Int. J. Trop Anim Health Prod., 29: 35-47. DOI: https://doi.org/10.1007/BF02632347

Jones A, Breuning-Madsen $\mathrm{H}$, Brossard M, Dampha A, Deckers J, Dewitte O, Gallali T, Hallett S, Jones R, Kilasara M, Le Roux P, Micheli E, Montanarella L, Spaargaren O, Thombiano L, Van Ranst E, Yemefack M, Zougmoré R. 2013. Soil Atlas of Africa. Union Européenne : Luxembourg.

Konaté F. 2008. Démographie - nvironnement Croissance démographique et systèmes de roduction au plateau dogon au Mali (edn). Tome, P.U.O., Cahiers du CERLESHS : Ouagadougou.

Kouadio KH, Ettien DJB, Bakayoko S, Soro D, Girardin O. 2014. Etude de la culture en couloirs de manioc (Manihot esculenta CRANTZ) à base de Gliricidia sepium en Côte d'Ivoire. Int J. Afrique Science, 10(3): 273-287.

Kuyah S, Whitney C, Jonsson M, Sileshi G.W, Öborn I, Muthuri C.W, Luedeling E. 2019. Agroforestry delivers a win-win solution for ecosystem services in sub-
Saharan Africa. Agron. Sustain Dev., 39: 47. DOI : https://doi.org/10.1007/s13593019-0589-8

Lahmar R, Bationo BA, Dan Lamso N, Guéro Y, Tittonell P. 2012. Tailoring conservation agriculture technologies to West Africa semi-arid zones: Building on traditional local practices for soil restoration. Int. J. Field Crops Res., 132: 158-167.

DOI: https://doi.org/10.1016/j.fcr.2011.09.013

Luedeling E, Smethurst PJ, Baudron F, Bayala J, Huth NI, van Noordwijk M, Ong C.K, Mulia R, Lusiana B, Muthuri C, Sinclair FL. 2016. Field-scale modeling of treecrop interactions: Challenges and development needs. Int. J. Agric. Syst., 142: 51-69. DOI: https://doi.org/10.1016/j.agsy.2015.11.00 5

Mafongaya PL, Kuntashula E, Kwesiga F, Chriwa T, Chintu R, Sileshi G, Matibini J. 2003. Leguminous Agroforestry Options for Replenishing Soil Fertility in Southern Africa. Grain Legumes and Green Manures for Soil Fertility in Southern Africa: Taking Stock of Progress. Soil Fert Net and CIMMYT: Harare.

Maraux F, Dugué P, Ganry F. 2007. Amélioration de la fertilité du sol et réhabilitation des terres dégradées : dynamiques socio-techniques en zones sèches d'Afriques de l'ouest et du centre. Accra (Ghana) : Agricultural innovation in dryland Africa: What are the key drivers for success ?, AIDA Conference, du 22 au 24 janvier 2007.

McBratney A, Field DJ, Koch A. 2014. The dimensions of soil security. Int. J. Geoderma., 213: 203-213. DOI: https://doi.org/10.1016/j.geoderma.2013. 08.013

Ouattara B, Coulibaly K, Kohio E, Doumbia S, Ouédraogo S, Nacro HB. 2018. Effets du Système de Culture sous couverture Végétale (SCV) sur les flux hydriques d'un sol ferrugineux à l'ouest du Burkina Faso. Int. J. Biol. Chem. Sci., 12(4): 1770- 
1783.

DOI:

https://dx.doi.org/10.4314/ijbcs.v12i4.20

Okonkwo CI, Mbagwu JSC, Egwu SO. 2009.

Changes in soil properties under alley cropping system of three leguminous crops. Journal of Tropical Agriculture, Food, Environment and Extension., 8(1) 60-65.

Partey ST, Zougmoré RB, Ouédraogo M, Campbell BM. 2018. Developing climate-smart agriculture to face climate variability in West Africa: Challenges and lessons learnt. Int. J. Cleaner Production., 187: 285-295. DOI: https://doi.org/10.1016/j.jclepro.2018.03. 199

Rosenstock T, Tully K, Arias-Navarro C, Neufeldt H, Butterbach-Bahl K, Verchot L. 2014. Agroforestry with N2-fixing tress: sustainable development's friend or foes? curr. Opin. Environ. Sustain., 6: 1521.

Sacande M, Sanogo S, Beentje H. 2016. Guide d'Identification des Arbres du Mali. Royal Botanic Gardens : Kew.

Smethurst PJ, Huth NI, Masikati P, Sileshi GW, Akinnifesi FK, Wilson J, Sinclair F. 2017. Accurate crop yield predictions from modelling tree-crop interactions in gliricidia-maize agroforestry. Int. J.
Agric. Syst., 155: 70-77. DOI: https://doi.org/10.1071/CP17311

Traoré L, Ouédraogo I, Ouédraogo A, Thiombiano A. 2011. Perceptions, usages et vulnérabilité des ressources végétales ligneuses dans le Sud-Ouest du Burkina Faso. Int. J. Biol. Chem. Sci., 5(1): 258278. DOI: 10.4314/ijbcs.v5i1.68103

Van der Wal A, de Boer W. 2017. Dinner in the dark: Illuminating drivers of soil organic matter decomposition. Int J. Soil Biology and Biochemistry, 105: 45-48. DOI: https://doi.org/10.1016/j.soilbio.2016.11. 006

Vanlauwe B, Aihou K, Tossah BK, Diels J, Sanginga N, Merckx R. 2005. Senna siamea trees recycle $\mathrm{Ca}$ from a Ca-rich subsoil and increase the topsoil $\mathrm{pH}$ in agroforestry systems in the West African derived savanna zone. Int. J. Plant Soil., 269: 285-296. DOI: https://doi.org/10.1007/s11104-0040599-3

Vanlauwe B, Kihara J, Chivenge P, Pypers P, Coe R, Six J. 2011. Agronomic use efficiency of $\mathrm{N}$ fertilizer in maize-based systems in sub-Saharan Africa within the context of integrated soil fertility management. Int. J. Plant Soil., 339: 3550. DOI: https://doi.org/10.1007/s11104010-0462-7. 